\title{
IHCb LHCb VELO Tracking Resolutions
}

\section{Impact Parameters}
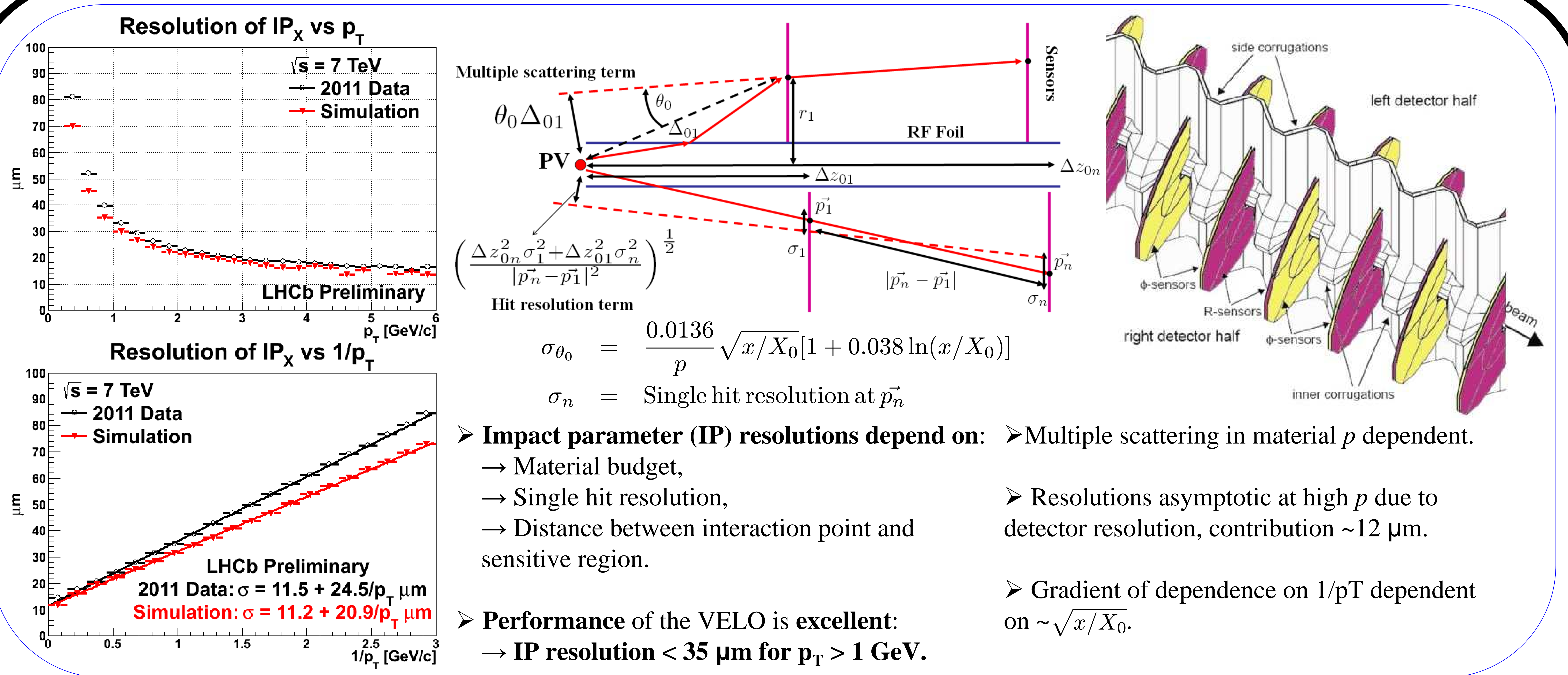

$>$ Impact parameter (IP) resolutions depend on: $>$ Multiple scattering in material $p$ dependent.

$\rightarrow$ Material budget,

$\rightarrow$ Single hit resolution,

$\rightarrow$ Distance between interaction point and

sensitive region.

$>$ Performance of the VELO is excellent:

$\rightarrow \mathrm{IP}$ resolution $<35 \mu \mathrm{m}$ for $\mathrm{p}_{\mathrm{T}}>1 \mathrm{GeV}$.

$>$ Resolutions asymptotic at high $p$ due to detector resolution, contribution $\sim 12 \mu \mathrm{m}$.

$>$ Gradient of dependence on $1 / \mathrm{pT}$ dependent on $\sim \sqrt{x / X_{0}}$.
$>$ Rotational alignment of VELO sensors \& modules about beam-line difficult to control using tracks from $\mathrm{p}-\mathrm{p}$ collisions.

$>$ Results in small sinusoidal bias to mean IP as a function of $\varphi$.

$>$ Rectified using tracks from beam-gas collisions that traverse the whole VELO.

$>$ Remaining bias negligible:

just few $\mu \mathrm{m}$.

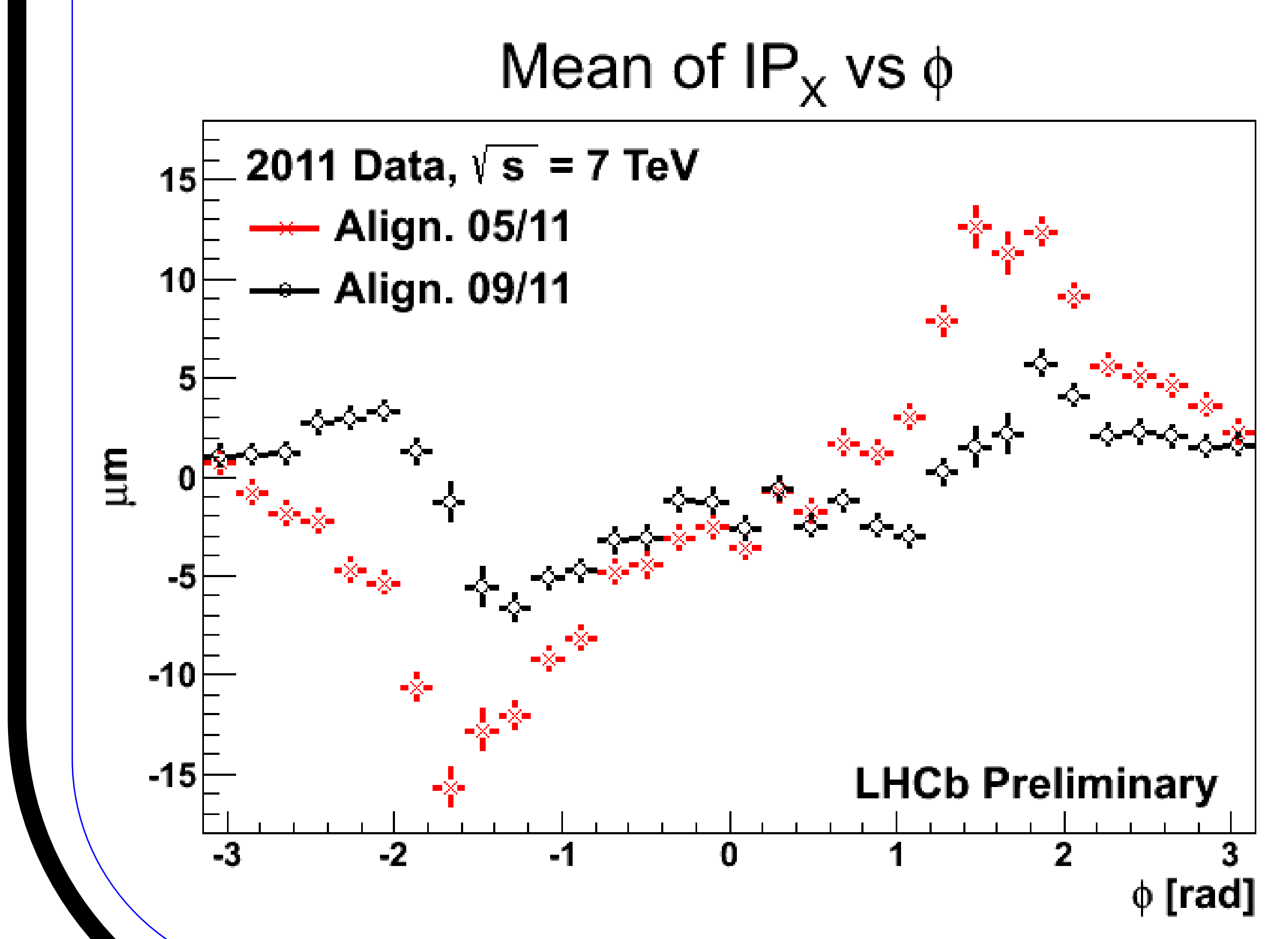

$>$ Compared to simulation IP resolutions on 2011 data very similar at high $\mathbf{p}_{\mathbf{T}}$ $>$ However, there is a large discrepancy at low $\mathbf{p}_{\mathbf{T}}-$ plotting vs $1 / \mathrm{p}_{\mathrm{T}}$ difference is only in the gradient.

$>$ Strongly suggests some detector material is missing from the simulation.

$>$ To get agreement would require huge amount of material to be added to simulation.

$>$ Pull studies show errors estimated correctly in overlap region, underestimated elsewhere.

$>$ However, detailed studies of VELO material have revealed no such glaring omission! $>$ The investigation continues..

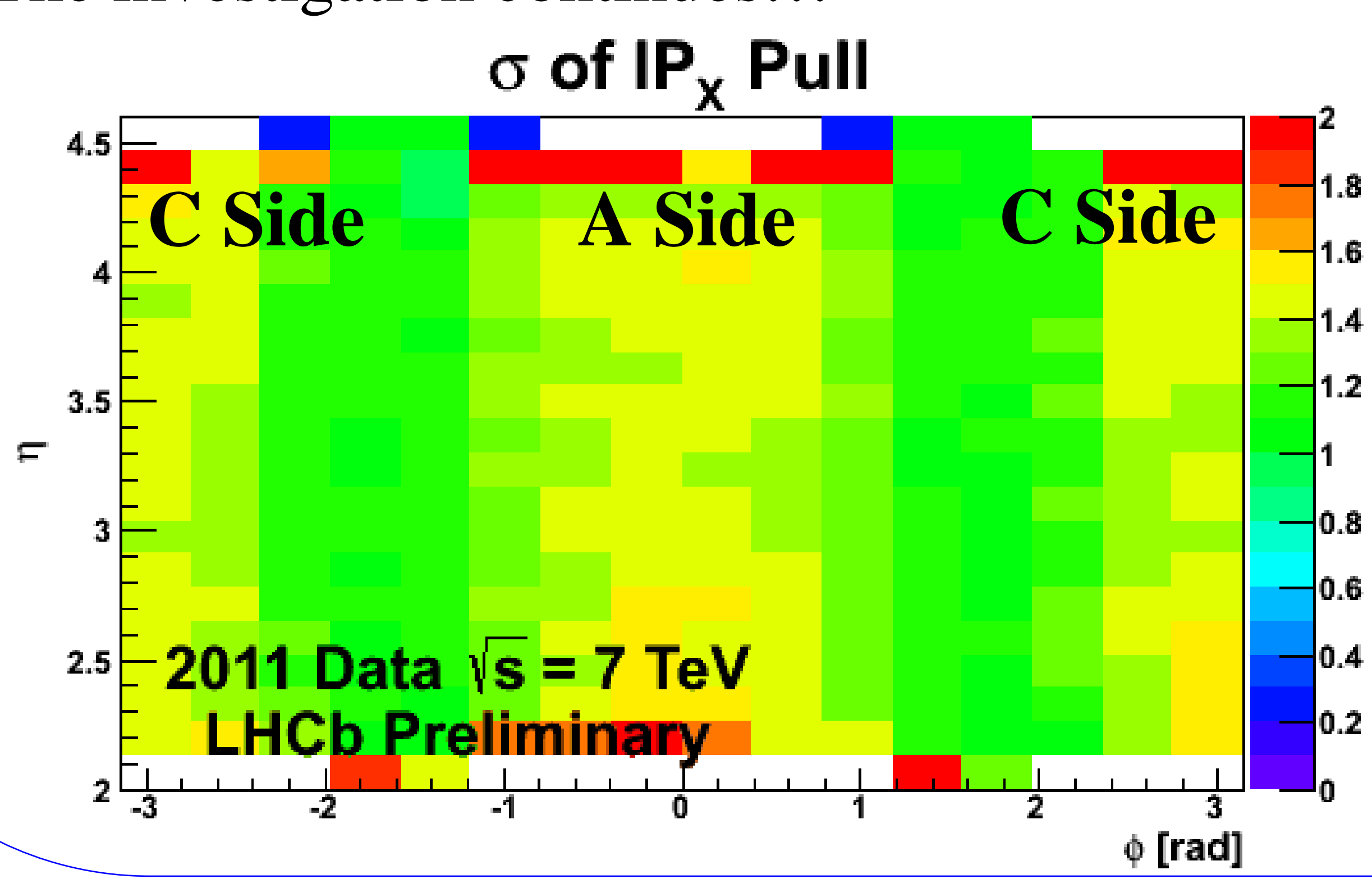

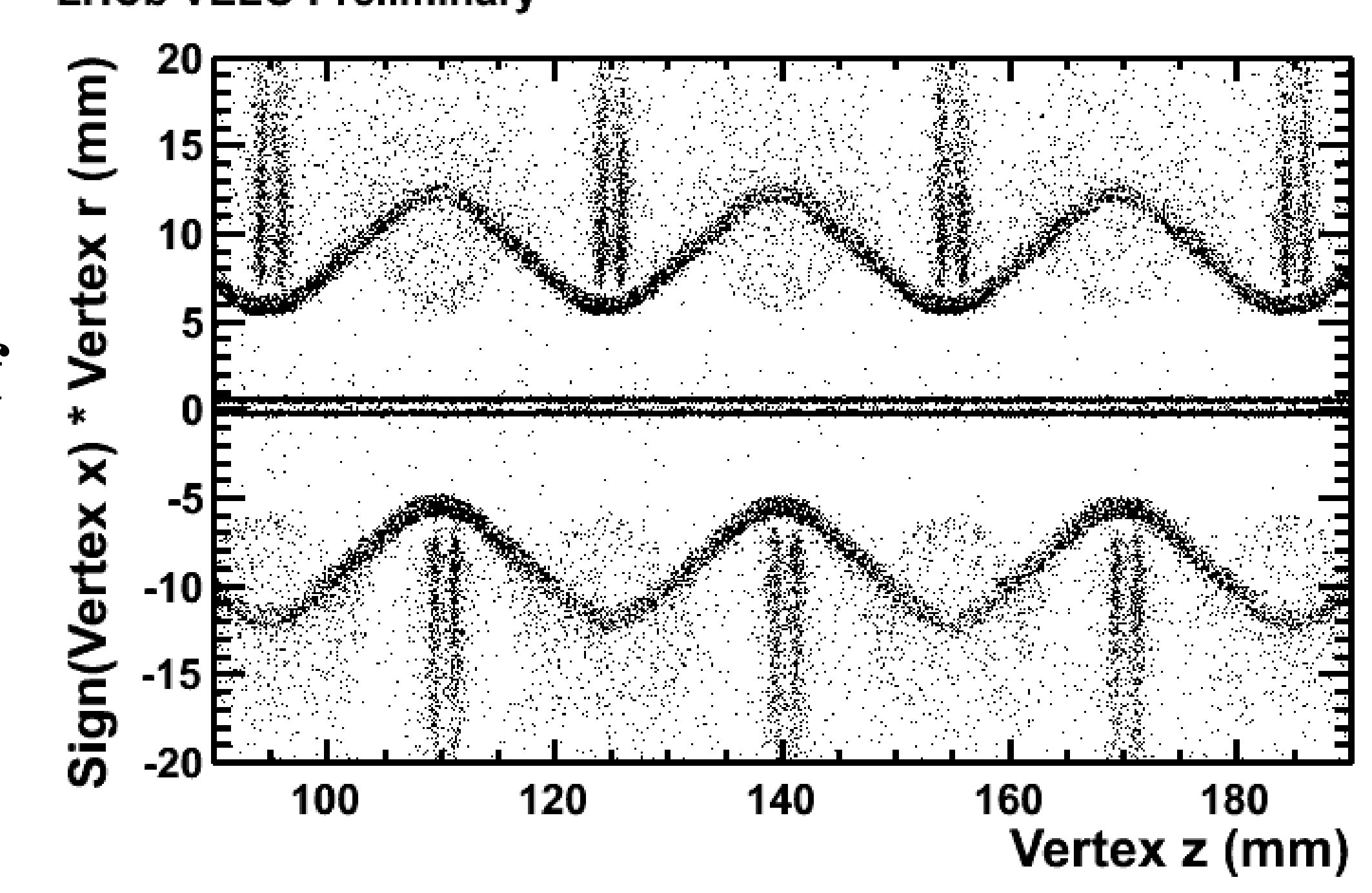

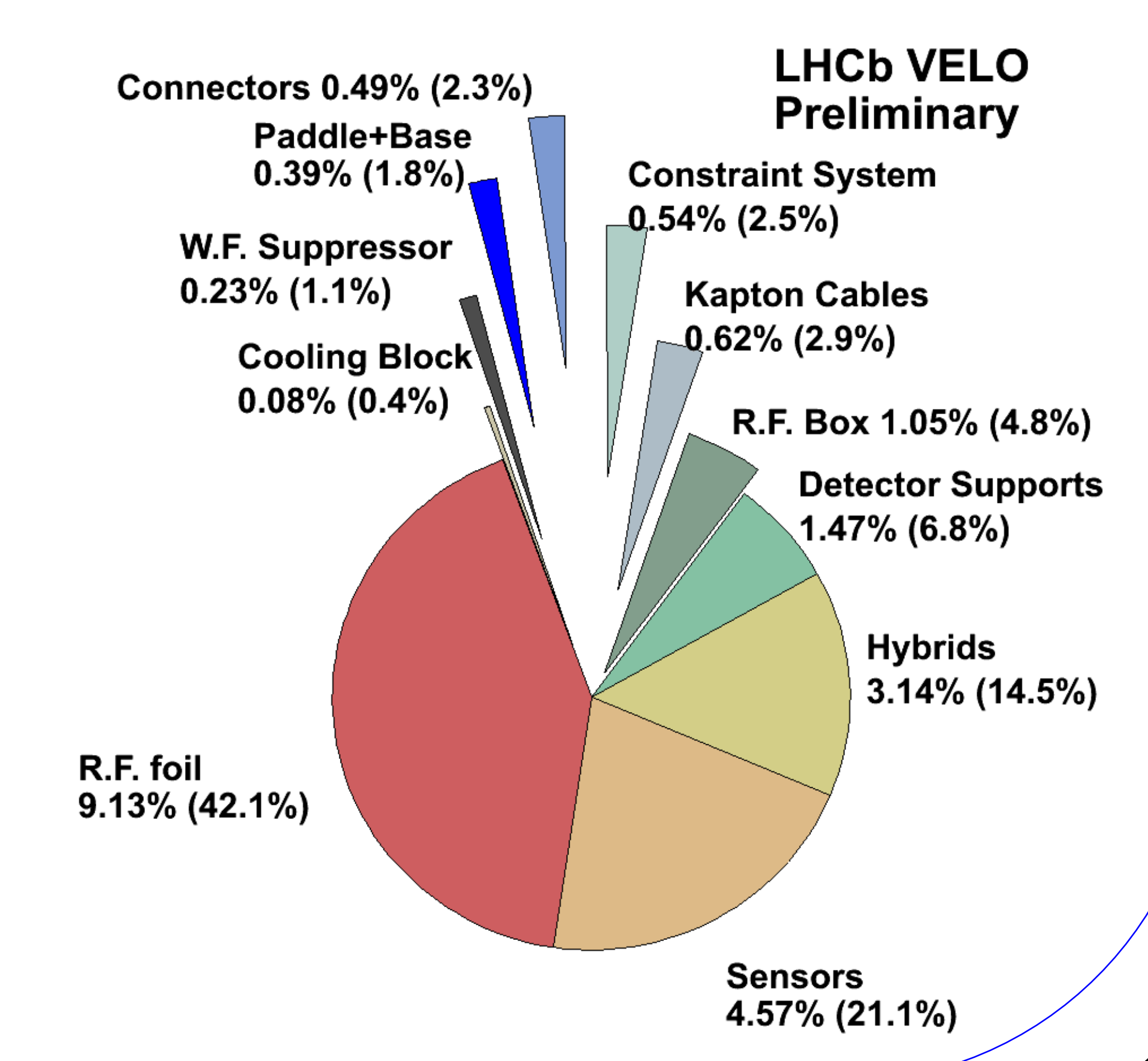

\section{Single Hits}

$>$ Best single hit resolution $4 \mu \mathrm{m}$ !

$>$ VELO very well designed for high precision measurements.

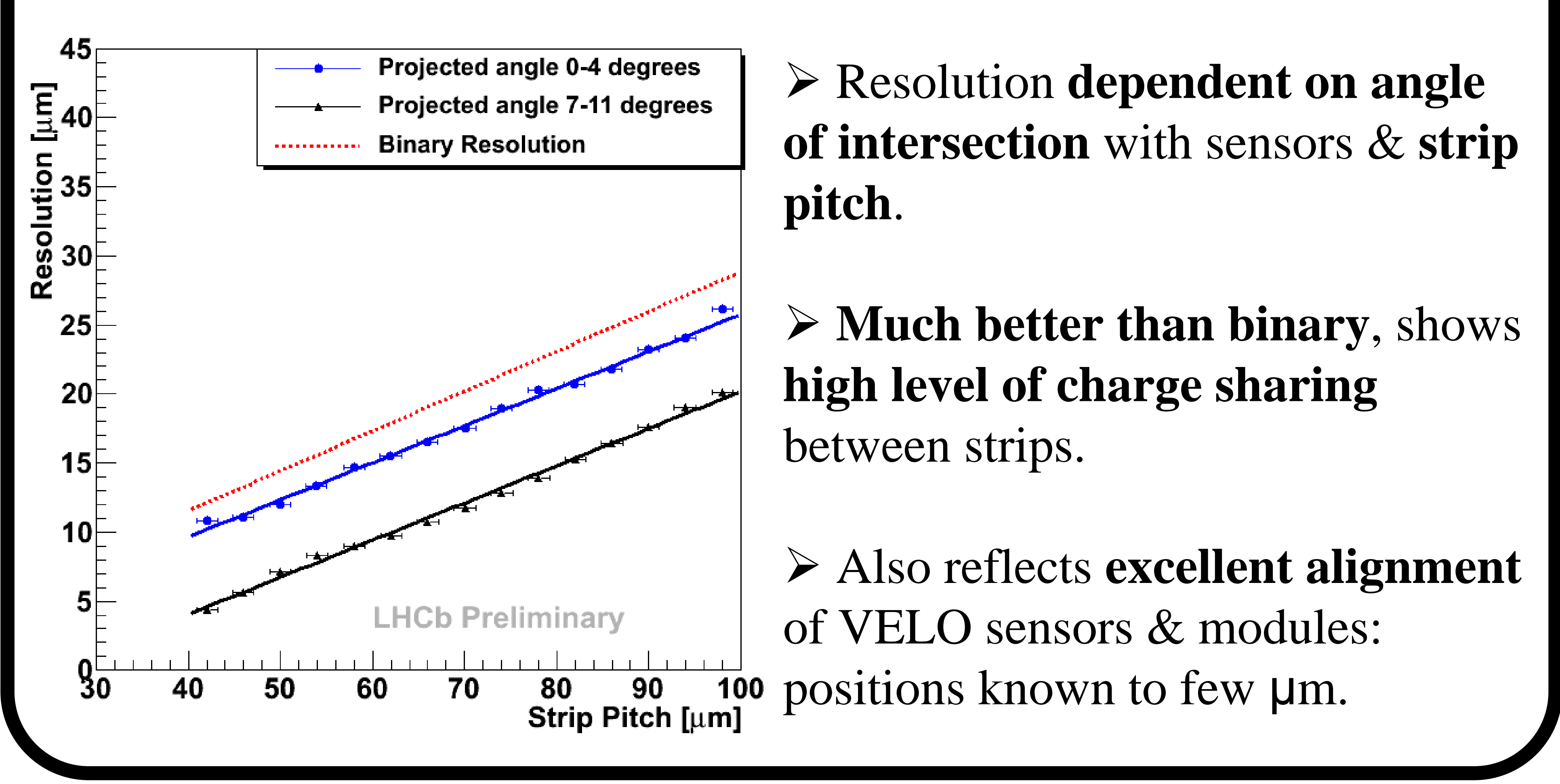

\section{Primary Vertices}

$>\mathrm{PV}$ resolution $\sim 13 \mu \mathrm{m}$

using 25 tracks.

$>$ Generally $50+$ tracks used for PVs in collision data.

$>$ Inversely dependent on N. tracks: fitted with $\mathrm{A} /\left(\mathrm{N}\right.$. tracks $^{\mathrm{B}}+\varepsilon$.

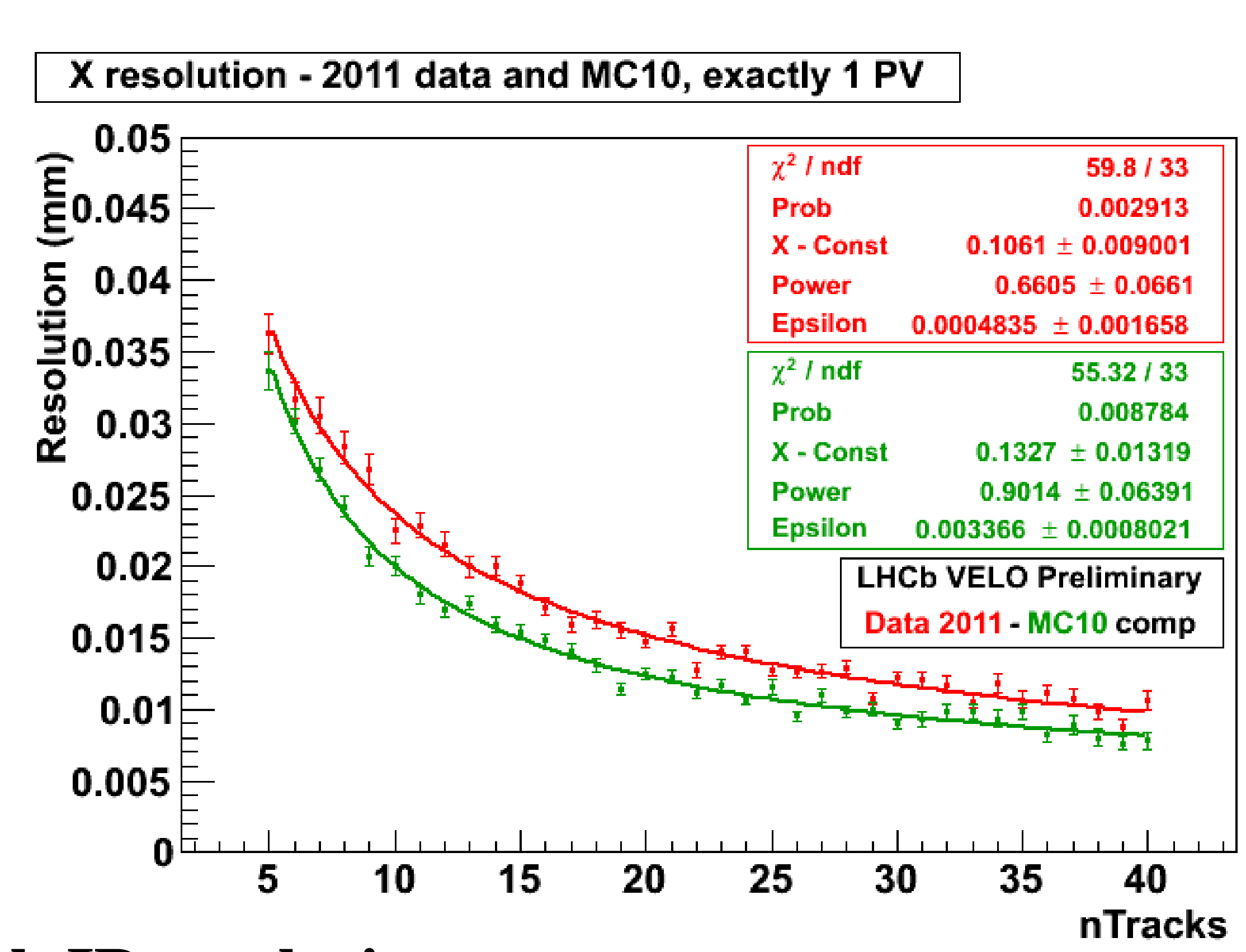

$>$ Strongly correlated with IP resolutions

> Similar $20 \%$ discrepancy between 2011 data \& simulation as IP resolution unsurprising. 